\title{
Multi-wavelength reflection spectra from an acousto-optic modulated fiber Bragg grating in a highly birefringent suspended core fiber
}

Ricardo E. Silva, Martin Becker, Manfred Rothhardt, Hartmut Bartelt, Alexandre A. P. Pohl

Ricardo E. Silva, Martin Becker, Manfred Rothhardt, Hartmut Bartelt, Alexandre A. P. Pohl, "Multi-wavelength reflection spectra from an acoustooptic modulated fiber Bragg grating in a highly birefringent suspended core fiber," Proc. SPIE 10323, 25th International Conference on Optical Fiber Sensors, 103238P (23 April 2017); doi: 10.1117/12.2265696

Event: 25th International Conference on Optical Fiber Sensors, 2017, Jeju, Korea, Republic of 


\title{
Multi-wavelength reflection spectra from an acousto-optic modulated fiber Bragg grating in a highly birefringent suspended core fiber \\ Ricardo E. Silva ${ }^{\mathrm{a}, \mathrm{b} *}$, Martin Becker ${ }^{\mathrm{a}}$, Manfred Rothhardt ${ }^{\mathrm{a}}$, Hartmut Bartelt ${ }^{\mathrm{a}}$ and Alexandre A. P. Pohl ${ }^{\mathrm{b}}$ \\ ${ }^{a}$ Leibniz Institute of Photonic Technology (IPHT), Albert-Einstein-Straße 9, Jena, Germany \\ ${ }^{b}$ Federal University of Technology-Paraná (UTFPR), Av.7 de Setembro 3165, Curitiba, Brazil
}

\begin{abstract}
The interaction of a fiber Bragg grating and longitudinal acoustic waves in a highly birefringent suspended-core fiber is investigated for the realization of a multi-wavelength reflection property. The modulated grating couples power from the fast and slow polarization modes to shifted superposed modes supported by the grating. The grating reflectivity of the superposed modes are tuned by the voltage of an electrical signal. Up to five different wavelength reflection peaks have been generated indicating new possibilities for compact and fast fiber-integrated multi-wavelength dynamic filters.
\end{abstract}

Keywords: Acousto-optic devices; microstructured fibers; fiber Bragg gratings.

\section{INTRODUCTION}

Fiber Bragg gratings (FBGs) in birefringent optical fibers have been successfully employed as multi-wavelength reflection filters in significant applications, such as, fiber sensors and fiber lasers [1-3]. In particular, fiber lasers with single or dual-wavelength being tuned by a polarization controller have been proposed [2]. Devices with two cascaded cavities including high-birefringent (Hi-Bi) gratings, have been used to achieve simultaneous four-wavelength laser output, by adjusting the polarization losses, the pump power and the fiber length [3]. A six-wavelength device using one grating in a few-mode Hi-Bi elliptical core fiber has also been reported [4].

An optical fiber with linear birefringence supports two polarization modes propagating in the core with distinct effective indices and phase velocities. However, anisotropies in the refractive index of the fibers can induce variations between the effective indices of the polarization modes, resulting in wavelength deviations. Additionally, external perturbations may change the grating properties in low birefringent fibers, inducing also changes in the reflected output power and wavelength. Although the influence of external perturbations is minimized for gratings inscribed in highbirefringent fibers, the use of conventional polarization controllers to switch the wavelength channels does not provide dynamic and fast reflectivity tuning of the reflected spectrum, since such devices usually are manually operated.

On the other hand, the modulation of Bragg gratings by means of longitudinal acoustic waves enables the electrical and fast tuning of the grating reflectivity and wavelength, which is suitable for optical modulators, dynamic filters and fiber lasers [5,6]. Figure 1(a) illustrates the reflection spectrum of a non-perturbed FBG of period $\Lambda$. The power of an optical mode with effective index $n_{\text {eff }}$ interacts with the grating being reflected at the Bragg wavelength $\lambda_{B}=2 n_{\text {eff }} \Lambda$. Figure 1(b) illustrates the acoustically induced strain modulating the grating period $\Lambda(z)$ along the fiber axis $z$, which induces reflection lobes on both sides of the Bragg resonance [5].

(a)

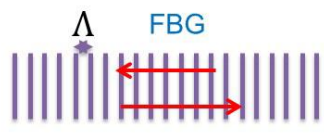

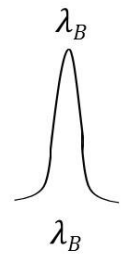

(b)

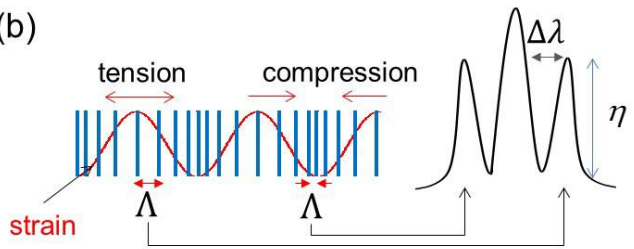

Figure 1. Illustration of the reflection spectrum of a fiber Bragg grating (a) without and (b) with modulation induced by a longitudinal acoustic wave.

*ricardoeqs@gmail.com

25th International Conference on Optical Fiber Sensors, edited by Youngjoo Chung, Wei Jin,

Byoungho Lee, John Canning, Kentaro Nakamura, Libo Yuan, Proc. of SPIE Vol. 10323

103238P - (C) 2017 SPIE · CCC code: 0277-786X/17/\$18 - doi: 10.1117/12.2265696 
In this way, the acoustic wave couples power from the Bragg resonance to higher order side lobes, modulating the lobe reflectivity $\eta$ and separation $\Delta \lambda$ by the acoustic power $P_{\text {ac }}$ and frequency $f$, respectively. Nevertheless, in a standard single-mode fiber (SMF), the acoustic power is uniformly distributed over the whole fiber cross section, reducing the overlap between the acoustic power and the grating in the core. One promising option to increase this acousto-optic interaction is the use of suspended-core fibers (SCFs) [7], which are generally composed of a very small core surrounded by a ring of large air holes. It reduces significantly the silica in the cladding, increasing the acousto-optic interaction in the core [8].

In this paper, an acoustically modulated Bragg grating inscribed in a highly birefringent double-core suspended-core fiber (HB-SCF) is experimentally investigated. The acoustically induced coupling from the orthogonal modes to superposed longitudinal modes of the grating is reported for the first time. The results indicate high acousto-optic modulation in such special HB-SCFs and new possibilities of dynamic filters and applications requiring multiwavelength spectral reflection properties.

\section{EXPERIMENTAL SETUP}

Figure 2(a) illustrates the setup used in the experiment. The acousto-optic modulator is composed of a piezoelectric transducer (PZT) disc (diameter of $25 \mathrm{~mm}$ and $2 \mathrm{~mm}$ in thickness), a $5 \mathrm{~cm}$ long acoustic silica horn and a $4.4 \mathrm{~cm}$ long highly birefringent double-core suspended-core fiber (HB-SCF) with the inscribed grating.

Figure 2(b) shows the cross section of the HB-SCF fabricated at IPHT, indicating the two similar birefringent cores. The fiber is composed of a rectangular pure silica region $(\sim 14 \mu \mathrm{m}$ length $\times 7.7 \mu \mathrm{m}$ width $)$, with a central air hole $(\sim 6 \mu \mathrm{m}$ length $\mathrm{x} 2 \mu \mathrm{m}$ width) surrounded by four air holes of $\phi \sim 40-43 \mu \mathrm{m}$ in diameter, separated by silica bridges of $t \sim 2.7 \mu \mathrm{m}$ thickness. The fiber diameter is $D \sim 124 \mu \mathrm{m}$.

We inscribed a $1 \mathrm{~cm}$ long FBG in the HB-SCF by means of a femtosecond laser and two-beam interference, using a phase mask interferometer according to the method described in [9]. The birefringence of the HB-SCF is estimated from the peaks of the fast and slow modes in the grating reflection spectrum as, $\Delta n_{\text {eff } \mathrm{XY}} \sim 2.2 \times 10^{-4}$.

The HB-SCF and the SMF connecting the circulator are aligned and the coupling power is monitored in transmission by means of a power meter. The SMF core is aligned only to one core of the HB-SCF, since the simultaneous power coupling to the two HB-SCF cores induces high insertion losses. The fibers are spliced by using an arc-discharge fusion splicer (Sumitomo F36).

The PZT is excited by a $1 \mathrm{~V}-10 \mathrm{~V}$ sinusoidal signal of a signal generator (SG) at the resonance of $f=874 \mathrm{kHz}$. The mechanical deformations of the transducer are amplified by the acoustic horn into longitudinal acoustic waves interacting with the grating.

A polarization controller (PC) is employed to align the polarization directions to the fast (Y), slow (X) and $\mathrm{XY}$ axes of the HB-SCF (XY indicates an angle of $45^{\circ}$ between the orthogonal polarization modes). The modulated spectrum is characterized with a wavelength resolution of $1 \mathrm{pm}$ for both polarizations by the Agilent 8164A Lightwave Measurement System, which is composed of a tunable laser diode (LD) and an integrated photodiode (FD).

(a)
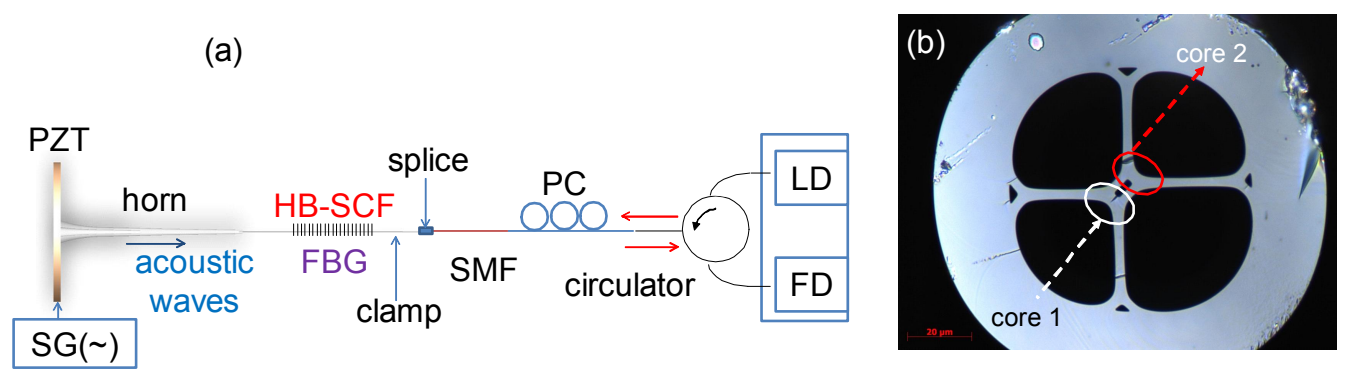

Figure 2. Illustration of the (a) experimental setup used to characterize the modulated FBG spectrum. (b) HB-SCF cross section 


\section{RESULTS AND DISCUSSION}

Figure 3 shows the FBG spectra for the polarizations $\mathrm{X}, \mathrm{Y}$ and $\mathrm{XY}$ with acoustic excitation at $f=874 \mathrm{kHz}$ in the voltage range of $1 \mathrm{~V}-10 \mathrm{~V}$. The data are normalized to the maximum grating reflectivity in linear scale. The acoustic wave induces reflection lobes on both sides of the fast and slow central modes. The reflectivity at the peaks $\lambda_{\mathrm{X}} \sim 1540.9 \mathrm{~nm}$ and $\lambda_{\mathrm{Y}} \sim 1540.7 \mathrm{~nm}$ is modulated similarly, achieving a maximum modulation depth of $\Delta \eta \sim 42 \%$ at $10 \mathrm{~V}$, if compared to the non-modulated grating. It indicates higher modulation efficiencies compared to previous studies employing standard single-mode fibers, considering similar resonances and modulation depth $\Delta \eta$ [10] (in this study, the $1 \mathrm{~cm}$ grating is $50 \%$ shorter and the maximum applied voltage is $\sim 75 \%$ lower).

The reflectivity of the left $\left(\lambda_{\mathrm{XC}}\right)$ and right $\left(\lambda_{\mathrm{YC}}\right)$ side lobes is indicated in Fig. 3(a-b). Figure 3(c) shows the modulated spectrum for the polarization XY (X and Y peaks are represented by $\left.\lambda_{\mathrm{XY}}\right)$. Note that the non-modulated grating has a low reflectivity superposed lobe centered at $\lambda_{\mathrm{XYC}} \sim 1540.8 \mathrm{~nm}$. The spectrum indicates that the central modes have the same effective indices and travel in phase at this wavelength. Thus, the acoustic wave induces side lobes coupling power from the fast and slow modes to the superposed central lobe. Here, we characterized just the longitudinal modes of the grating.

Figure 3(d) shows the comparison of the modulated spectra for both polarizations at $10 \mathrm{~V}$. The frequency is intentionally tuned at $f=874 \mathrm{kHz}$ to match the inner side lobes at the coupling wavelength $\lambda_{\mathrm{XYC}}$. Note that the peaks of the fast, slow and superposed modes achieve the same reflectivity at about $10 \mathrm{~V}$, with a maximum modulation depth of $\Delta \eta_{\mathrm{XYC}} \sim 16 \%$. This value is limited by the maximum $10 \mathrm{~V}$ of the signal generator. However, it is expected that higher voltages can couple a higher level of power to the superposed lobe. The side lobe separation $\Delta \lambda$ and wavelength sweep resolution are limited in this study due to the discrete behavior of the resonances for the employed PZT.

The modulation voltage responses for both polarization modes are summarized in Fig. 4, indicating the maximum modulation depth $\Delta \eta$ at the peaks $\lambda_{\mathrm{X}}$ and $\lambda_{\mathrm{Y}}$, and the induced side lobes $\lambda_{\mathrm{XC}}$ and $\lambda_{\mathrm{YC}}$ (Fig. 4(a)) and, at the peaks of the $\mathrm{XY}$ polarization $\lambda_{\mathrm{XY}}$ and the central lobe $\lambda_{\mathrm{XYC}}$ (Fig. 4(b)). Note in Fig. 4(b), that a maximum reflectivity superposition of $\Delta \eta_{\mathrm{XYC}} / \Delta \eta_{\mathrm{XY}} \sim 100 \%$ is achieved, indicating almost total coupling from the orthogonal modes to the superposed mode. The results indicate that the acoustically induced overlapping depends on the side lobe tuning to the coupling wavelength. However, a partial side lobe overlapping might be useful to adjust the reflectivity of the central, left and right side lobes to the same level. In this case, a voltage increase above $10 \mathrm{~V}$ may induce five flat wavelengths.
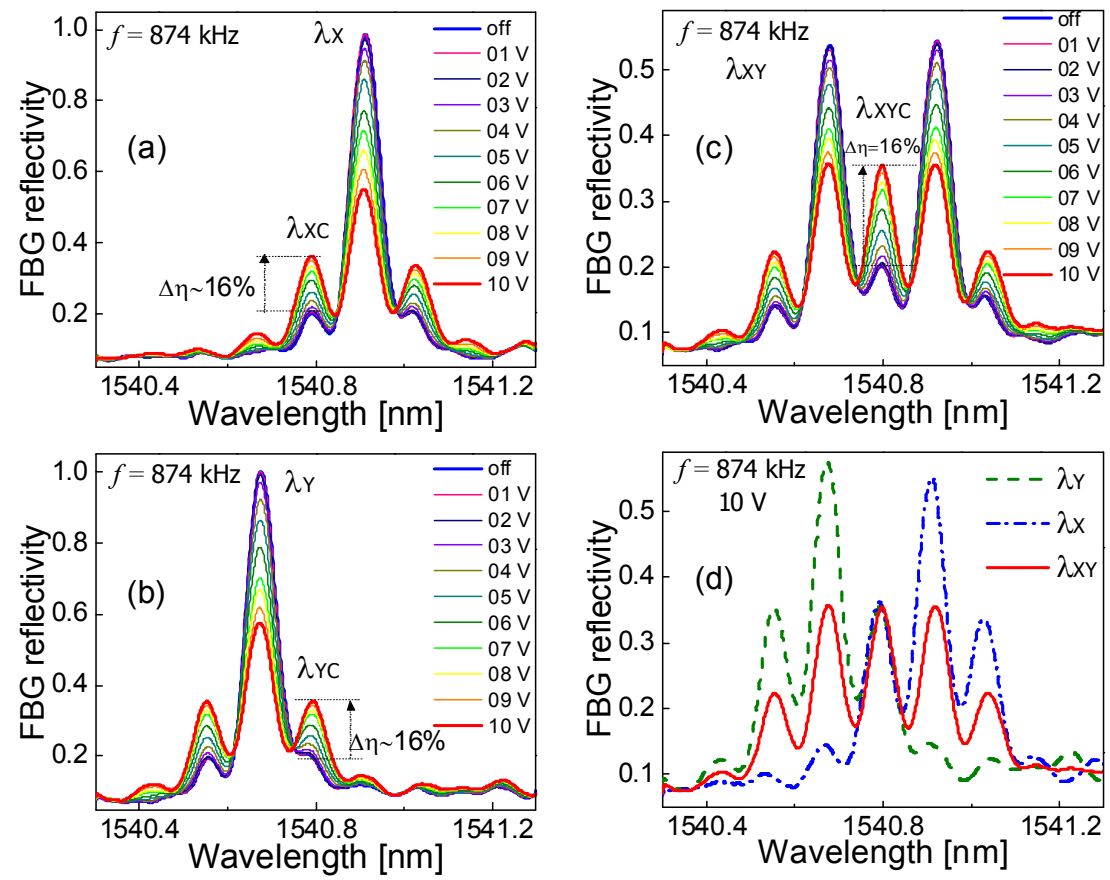

Figure 3. FBG modulated spectra for the polarizations (a) X, (b) $\mathrm{Y}$ and (c) $\mathrm{XY}$ at $f=874 \mathrm{kHz}$ and the voltage range of $1 \mathrm{~V}-10 \mathrm{~V}$ applied to the PZT. (d) Comparison of the modulated spectra for both polarizations at $10 \mathrm{~V}$. 

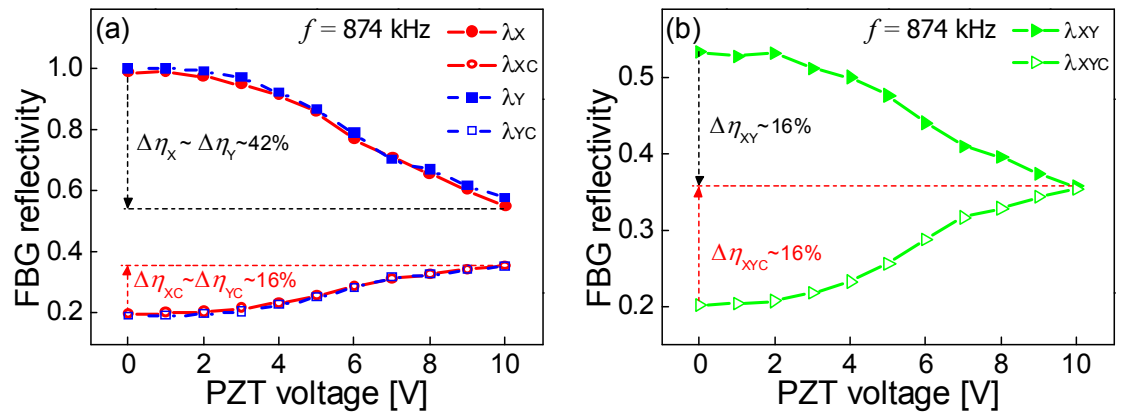

Figure 4. FBG modulation voltage response for the polarizations (a) $\mathrm{X}, \mathrm{Y}$ and (b) $\mathrm{XY}$ at the resonance of $f=874 \mathrm{kHz}$ and the voltage range of $1 \mathrm{~V}-10 \mathrm{~V}$.

In summary, the coupling from the orthogonal modes to superposed modes in a highly birefringent suspended-core fiber by an acoustically modulated Bragg grating in combination with a polarization controller provides the possibility to achieve a reflection spectrum with up to 5 discrete wavelengths, whose reflectivity level can be tuned by an electrical signal. It expected that this number of channels might be increased to 10 with the same fiber considering the simultaneous modulation of the two cores in the fiber. In addition, the use of a $1 \mathrm{~cm}$ grating in a fiber with large air holes points to higher acousto-optic modulation compared to previous studies employing solid standard fibers, which is suitable to reduce the fiber/grating length, the size and the acoustic power of the modulator for the multi-wavelength reflection characteristics.

\section{ACKNOWLEDGMENT}

This work was supported in part by the Cordenação de Aperfeiçoamento de Pessoal de Nível Superior (CAPES) and by the Fundação Araucária de Apoio ao Desenvolvimento Científico e Tecnológico do Estado do Paraná, Brazil. Funding by the Thuringian Ministry of Education, Science and Culture (EFRE program), Germany, is gratefully acknowledged.

\section{REFERENCES}

[1] Chen, G., Liu, L., Jia, H., J., Xu, L. and Wang, W., "Simultaneous strain and temperature measurements with fiber Bragg grating written in novel Hi-Bi optical fiber," IEEE Photonics Technol. Lett. 16, 221-223 (2004).

[2] Hernandez-Cordero, J., Kozlov, V. A., Carter, A. L. G., and Morse, T. F., "Fiber laser polarization tuning using a Bragg grating in a Hi-Bi fiber," IEEE Photonics Technol. Lett. 10, 941-943 (1998).

[3] Liu, Y., Feng, X., Yuan, S., Kai, G., and Dong, X., "Simultaneous four-wavelength lasing oscillations in an erbiumdoped fiber laser with two high birefringence fiber Bragg gratings," Opt. Express 12, 2056 (2004).

[4] Moon, D. S., Paek, U.C., and Chung, Y., "Polarization controlled multi-wavelength Er-doped fiber laser using fiber Bragg grating written in few-mode side-hole fiber with an elliptical core," Opt. Express 13, 5574 (2005).

[5] Liu, W. F., Russell, P. S. J., and Dong, L., "100\% efficient narrow-band acoustooptic tunable reflector using fiber Bragg grating," J. Light. Technol. 16, 2006-2009 (1998).

[6] Silva, R. E., Tiess, T., Becker, M., Eschrich, T., Rothhardt, M., Jäger, M., Pohl, A. A. P. and Bartelt, H., "Acoustooptic modulation of a fiber Bragg grating in suspended core fiber for mode-locked all-fiber lasers," Laser Phys. Lett. 12, 45101 (2015).

[7] Phan Huy, M. C., Laffont, G., Dewynter, V., Ferdinand, P., Roy P., Auguste, J. L., Pagnoux, D., Blanc, W., and Dussardier, B., "Three-hole microstructured optical fiber for efficient fiber Bragg grating refractometer," Opt. Lett. 32, 2390 (2007).

[8] Silva, R. E., Hartung, A., Rothhardt, M., Pohl, A. A. P., and Bartelt, H., "Detailed numerical investigation of the interaction of longitudinal acoustic waves with fiber Bragg gratings in suspended-core fibers," Opt. Commun. 344, 43-50 (2015).

[9] Becker, M., Bergmann, J., Brückner, S., Franke, M., Lindner, E., Rothhardt, M., and Bartelt, H., "Fiber Bragg grating inscription combining DUV sub-picosecond laser pulses and two-beam interferometry," Opt. Express 16, 19169 (2008).

[10] Oliveira, R. A., Neves Jr., P. T., Pereira, J. T., Canning, J. and Pohl, A. A. P., "Vibration mode analysis of a silica horn-fiber Bragg grating device," Opt. Commun. 283, 1296-1302 (2010). 